\title{
NF-кB upregulates ubiquitin C-terminal hydrolase 1 in diseased podocytes in glomerulonephritis
}

\author{
HONGXIAZHANG ${ }^{1,2}$, XING MAO ${ }^{2}$, YU SUN ${ }^{2}$, RUIMIN HU ${ }^{2}$, WEILI LUO ${ }^{2}$, \\ ZHONGHUA ZHAO $^{2}$, QI CHEN ${ }^{2}$ and ZHIGANG ZHANG ${ }^{2}$ \\ ${ }^{1}$ Department of Pathology, Weifang Medical College, Weifang, Shandong 261053; \\ ${ }^{2}$ Department of Pathology and Key Laboratory of Molecular Medicine (Ministry of Education), \\ College of Basic Medicine, Fudan University, Shanghai 200032, P.R. China
}

Received July 23, 2014; Accepted April 10, 2015

DOI: $10.3892 / \mathrm{mmr} .2015 .3780$

\begin{abstract}
Podocyte injury is a pivotal factor during the progression of glomerular diseases. It has been demonstrated that the expression of ubiquitin carboxy-terminal hydrolase L1 (UCH-L1) is increased in injured podocytes in a number of types of glomerulonephritis. However, its mechanism of regulation remains to be elucidated. A previous study by our group suggested that UCH-L1 is a downstream protein of nuclear factor (NF)- $\mathrm{kB}$ signaling. In the present study, the involvement of NF- $\mathrm{kB}$ in the regulation of the expression of UCH-L1 was investigated in diseased podocytes in vivo and in vitro. Increases in the expression of phosphorylated NF- $\mathrm{kB}$ at $\mathrm{p} 65$ and UCH-L1 were detected using immunohistochemical analysis of kidney biopsy tissues from 56 cases of nephritis, including immunoglobulin A nephropathy, membranous glomerulonephritis and lupus nephritis. The two indicators were also analyzed using western blot analysis in cultured murine podocytes stimulated by inflammatory factors. The results of the present study demonstrated that in human renal biopsies of several cases of immune complex-mediated glomerulonephritis, the increases of NF- $\mathrm{KB}$ and UCH-L1 were positively correlated with the number of diseased podocytes. By contrast, in non-immune complex-mediated glomerulonephritis, no clear activation of NF- $\mathrm{kB}$ and increase of UCH-L1 expression was observed. In vitro, immune stimulation also led to the upregulation of UCH-L1 through the NF- $\mathrm{KB}$ signaling pathway in mouse podocytes. In conclusion, the results of the present study suggested that the activation of NF- $\mathrm{KB}$ and upregulation of UCH-L1 in podocytes may be vital in
\end{abstract}

Correspondence to: Professor Zhigang Zhang, Department of Pathology and Key Laboratory of Molecular Medicine (Ministry of Education), College of Basic Medicine, Fudan University, 138 Yixueyuan Road, Shanghai 200032, P.R. China

E-mail: zzg@shmu.edu.cn

Key words: podocyte, ubiquitin carboxy-terminal hydrolase L1, nuclear factor- $\kappa \mathrm{B}$, immune complex-mediated nephritis podocyte injury associated with immune complex-mediated glomerulonephritis.

\section{Introduction}

The podocyte is one of the resident cells of the glomerulus, which is often attacked as the target cell in the glomerulus by a variety of immune or non-immune inflammatory factors. Podocytopathy refers to the various degrees of podocyte injury occurring in pathological conditions. Due to their highly differentiated state and limited proliferative capability, podocytes are not able to recover from damage effectively. Therefore, podocyte injury is a pivotal factor during the progression of glomerular diseases (1-3).

During the disease process of glomerulonephritis, numerous interconnected network signaling cascades are activated in renal cells. The mammalian nuclear factor $(\mathrm{NF})-\kappa \mathrm{B}$ signaling pathway, which is a fundamental intracellular transcription factor system, is induced in response to various sources of extracellular stimulation. An indicator of $\mathrm{NF}-\kappa \mathrm{B}$ activation is the nuclear translocation of dimeric Rel protein 8 , which regulates numerous NF- $\mathrm{BB}$-dependent genes involved in inflammation, immunity, apoptosis, cell proliferation and differentiation (4-6). Accumulating lines of evidence have indicated that the activation of $\mathrm{NF}-\kappa \mathrm{B}$ is a critical response to kidney diseases. Upregulation of the canonical (RelA/p50) $\mathrm{NF}-\kappa \mathrm{B}$ isoform in macrophages, mesangial cells, tubular epithelial cells and podocytes has a pathogenic role in mediating acute and chronic inflammatory nephropathy (7-13). A number of in vivo studies of podocytes in nephrotic glomerular diseases have demonstrated that NF- $\kappa \mathrm{B}$ and RelA are markedly upregulated. For example, $N F-\kappa B$ is activated within podocytes in passive Heymann nephritis, which contributes to autologous phase proteinuria (14). Furthermore, in patients with Lupus nephritis (LN), activation of NF- $\mathrm{NB}$ (predominantly at p65) and upregulation of interleukin (IL)-1, IL-4 and tumor necrosis factor (TNF)- $\alpha$ are co-localized in diseased podocytes. The staining score of activated NF- $\kappa$ B p65 has been observed to be positively correlated with the severity of proteinuria (11). Treatment with genistein, which is able to inhibit pro-inflammatory cytokines through downregulation of the NF- $\mathrm{NB}$ pathway, prevented pathological changes in 
podocytes, including extensive disruption and effacement of processes (13). In vitro, $\mathrm{NF}-\kappa \mathrm{B}$ is activated in podocytes in response to their exposure to various factors, which may cause kidney damage. For example, $\mathrm{NF}-\kappa \mathrm{B}$ was upregulated in murine podocytes exposed to either a Shiga toxin or protein overload, and mediated the increase of endothelin-1 production $(15,16)$. Angiotensin II is capable of upregulating the expression of Toll-like receptor 4 in murine podocytes, which may effectively lead to NF- $\kappa \mathrm{B}$ activation (17). Transforming growth factor (TGF)- $\beta 1$ may induce podocyte damage by upregulating transient receptor potential cation channel, subfamily $\mathrm{C}$, member 6 protein, most likely through the Smad3-extracellular signal-regulated kinase-NF- $\mathrm{B}$ pathway (18). Therefore, it was suggested that the podocyte injury was induced mainly via $\mathrm{NF}-\kappa \mathrm{B}$ activation, which may further mediate a large number of NF- $\kappa \mathrm{B}$ target genes and cause morphological and functional abnormalities of podocytes.

A previous in vitro study by our group demonstrated that ubiquitin carboxy-terminal hydrolase L1 (UCH-L1) is a downstream target protein of $\mathrm{NF}-\kappa \mathrm{B}$. The activation of $\mathrm{NF}-\kappa \mathrm{B}$ by inflammatory factors may increase the expression of UCH-L1 in murine podocytes and affect morphological changes in podoctyes (19).

$\mathrm{UCH}-\mathrm{L} 1$ is a member of the deubiquitinating enzyme family, which is important in the regulation of the ubiquitin-proteasome system $(20,21)$. Although it is mainly localized to the brain and testis, UCH-L1 is also expressed in the kidney and involved in nephrogenesis (22-24). Previous studies by our group and others reported that UCH-L1 is involved in podocyte differentiation and injury. The level of expression was increased in podocytes in a variety cases of immune complex-mediated glomerulonephritis $(1,2,25)$. In cultured HEK293T cells, using a luciferase assay, it was further demonstrated that $\mathrm{NF}-\kappa \mathrm{B}$ upregulates $\mathrm{UCH}-\mathrm{L} 1$ via binding to the $-300 \mathrm{bp}$ and $-109 \mathrm{bp}$ sites of the UCH-L1 promoter. This supported the hypothesis that UCH-L1 was upregulated in podocytes through the NF- $\mathrm{KB}$ signaling pathways (19).

The present study further investigated the involvement of $\mathrm{NF}-\kappa \mathrm{B}$ in the regulation of expression of UCH-L1 in podocytes under inflammatory conditions. Expression levels of $\mathrm{NF}-\kappa \mathrm{B}$ and $\mathrm{UCH}-\mathrm{L} 1$ were detected using immunohistochemistry in kidney biopsy tissues from specific forms of immune complex-mediated glomerulonephritis, including LN, immunoglobulin A nephropathy (IgAN) and membranous glomerulonephritis (MGN). These proteins were also assessed in vivo in murine podocytes co-cultured with mesangial cells and treated with rabbit anti-rat thymocyte serum (ATS), which may interact with mesangial cells to form immune complexes.

\section{Patients and methods}

Immunohistochemistry. Tissues from 56 individuals, including 1 normal control, 19 patients with LN, 15 patients with IgAN, 15 patients with MGN and 6 patients with minimal change disease (MCD), were collected from renal needle biopsies from the Nephrosis Laboratory, Department of Pathology, (Fudan University, Shanghai, China) between 2010 and 2013. Permission to use the tissue sections for research purposes was obtained and approved by the Ethics Committee from the College of Basic Medicine, Fudan University, and a written consent form was obtained from all patients. Sections (4 $\mu \mathrm{m}$ ) were deparaffinized in $100 \%$ xylene for $10 \mathrm{~min}$ then rehydrated gradually in an alcohol series. They were then incubated in a $0.3 \%$ hydrogen peroxide/methanol buffer for $30 \mathrm{~min}$ to quench endogenous peroxidase activity. Antigen retrieval was performed by immersing the sections in $0.5 \mathrm{~mol} / \mathrm{l}$ ethylenediaminetetraacetic acid buffer $(\mathrm{pH} 8.0)$ for $3 \mathrm{~min}$, followed by boiling in a water bath for $7 \mathrm{~min}$. The sections were rinsed in phosphate-buffered saline (PBS) and subsequently incubated with rabbit polyclonal anti-UCH-L1 antibody (1:100; cat. no. AB1761; Millipore, Billerica, MA, USA) overnight at $4^{\circ} \mathrm{C}$ in a humidified chamber. Following incubation, the sections were washed three times with PBS containing $0.05 \%$ Tween-20 for 5 min each time. The sections were then incubated with $100 \mu \mathrm{l}$ horseradish peroxidase conjugated-anti-rabbit immunoglobulin $\mathrm{G}$, maintaining the tissue section in a moist chamber for $30 \mathrm{~min}$ at $37^{\circ} \mathrm{C}$ and then the sections were washed three times, as previously. Immobilized antibodies were detected using a two step EnVision+ system peroxidase kit (Dako, Carpinteria, CA, USA). 3,3'-diaminobenzidine was used as the chromogen and hematoxylin as the nuclear counterstain (Sangon Biotech Co., Ltd., Shanghai, China). The sections were rinsed thoroughly with tap water for 1-2 min, then they were rinsed with distilled water, followed by tap water. The protocol for assessing activated NF-кB p65 (rabbit monoclonal anti-p-p65 antibody; 1:100; cat. no. 3033; Cell Signaling Technology, Beverly, MA, USA) and Wilms tumor 1 (WT1; rabbit polyclonal anti-WT1 antibody; 1:100; cat. no. P-0526; Changdao Biotech Co., Ltd., Shanghai, China) in successive sections was similar to that described above. The positive cell number of all slides was counted by two individuals.

Cell culture. The conditionally thermosensitive SV40-transfected immortalized murine podocyte cell line MPC5 (a gift from Professor Xu Hong; Affiliated Children's Hospital of the Medical College of Fudan University, Shanghai, China) was cultured as described previously (19). Briefly, MPC5 cells were cultured under permissive conditions $\left[33^{\circ} \mathrm{C}, 5 \%(\mathrm{v} / \mathrm{v})\right.$ $\mathrm{CO}_{2}$, RPMI-1640 (Gibco Life Technologies, Carlsbad, CA, USA), $10 \%$ (v/v) fetal bovine serum (Gibco Life Technologies), 50-10 U/ml $\gamma$-interferon (ProSpec-Tany TechnoGene Ltd., Ness Ziona, Israel)] and under formulary conditions $\left(37^{\circ} \mathrm{C}\right.$ without $\gamma$-interferon) for external differentiation. Podocytes, which were between passages 5 and 20 were used. Differentiated cells were identified by their large arborized shape and by cell expression of synaptopodin mRNA, a known marker for differentiation. An inverted microscope (TS100; Nikon, Tokyo, Japan) was used to observe the growth of podocytes.

Reverse transcription-quantitative polymerase chain reaction (RT-qPCR). Synaptopodin mRNA expression was identified using synaptopodin DNA. Total RNA from differentiated and non-differentiated podocyte cells was isolated using the RNAiso Plus extraction reagent and cDNA was produced using PrimeScript reverse transcriptase (Takara Bio, Inc., Otsu, Japan) according to the manufacturer's instructions. RT-qPCR was then conducted using SYBR Premix Ex Taq II (Takara Bio, Inc.) in $25 \mu \mathrm{l}$ reactions with $2 \mu \mathrm{l}$ cDNA and $0.4 \mu \mathrm{M}$ each 
of the forward and reverse primers using the following thermocycling conditions: $95^{\circ} \mathrm{C}$ for $30 \mathrm{sec}, 95^{\circ} \mathrm{C}$ for $5 \mathrm{sec}$ and $60^{\circ} \mathrm{C}$ for $30 \mathrm{sec}$ for 40 cycles. Data was collected and all expression levels were normalized to $\beta$-actin. The ABI7900 thermocycler (Life Technologies, Carlsbad, CA, USA) was used. The following primers were used: Synaptopodin, forward 5'-CCT GCCCGTAACTTCCGTG-3', and reverse 5'-GAGCGGCGG TAGGGAAAAG-3'; $\beta$-actin, forward 5'-CATCCGTAAAGA CCTCTATGCCAAC-3', and reverse 5'-ATGGAGCCACCG ATCCACA-3'. The primers were synthesized by Jie Li Biology (Shanghai, China).

Subsequently, the cells were treated with TNF- $\alpha$ (15 ng/ml; Sigma-Aldrich, St. Louis, MO, USA), IL-1 $\beta$ (15 ng/ml; Sigma-Aldrich) and lipopolysaccharide (LPS; $1 \mu \mathrm{g} / \mathrm{ml}$; Sigma-Aldrich) and ATS $(50 \mu \mathrm{l} / \mathrm{ml})+$ human serum $(30 \mu \mathrm{l} / \mathrm{ml})$ for the indicated durations. Pyrrolidine dithiocarbamate (PDTC; Sigma-Aldrich) was used to treat podocytes at various concentrations for $2 \mathrm{~h}$ before the cells were treated with TNF- $\alpha$, IL- $1 \beta$, LPS and ATS + human serum for $24 \mathrm{~h}$.

In the podocyte-mesangial cell co-culturing system, podocytes at $80 \%$ confluence were co-cultured with mesangial cells [prepared from the kidney cortex of male Sprague-Dawley rats as described previously (26) and planted in nested transwell plates (Corning Inc., Corning, NY, USA) at 30\% confluence] in six-well plates. Sprague-Dawley rats were obtained from the Animal Center of the College of Basic Medicine of Fudan University). A total of 10 rats at 2 months-old were used in the present study. The protocol was approved by the Ethics Committee from the College of Basic Medicine, Fudan University. Subsequently, the dual culture system was treated with ATS $>1: 1,000$ for different time periods.

The ATS was produced in our laboratory (27). It is able to combine with antigens of rat mesangial cells and activate complement in the serum, and the resulting immune complex is capable of stimulating podocytes directly in the co-culture system. The ATS was verified to be efficient at a dilution of 1:1,000 using the agarose immunodiffusion method as described previously (27).

Western blot analysis. Gel electrophoresis and western blotting were performed as described previously (19). Samples $(15 \mu \mathrm{l})$ of the podocyte lysates, including soluble cell proteins, were separated by SDS-PAGE $[10 \%(\mathrm{w} / \mathrm{v})$ polyacrylamide gel; Bio-Rad Laboratories, Inc., Hercules, CA, USA] and then transferred electrophoretically onto polyvinylidene difluoride membranes (Millipore). The membranes were blocked with $5 \%(\mathrm{v} / \mathrm{v})$ nonfat milk and probed with primary antibodies against UCH-L1 (Millipore), p65/p-p65 (1:2,000; cat. no. 4764; Cell Signaling Technology) and $\beta$-actin (as a loading control) overnight at $4^{\circ} \mathrm{C}$ following incubation with horseradish peroxidase-conjugated secondary antibody (Proteintech Group, Chicago, IL, USA). All washing steps were performed in Tris-buffered saline containing $0.1 \%(\mathrm{v} / \mathrm{v})$ Tween 20 (Sangon Biotech Co., Ltd.). The immune reaction was visualized via an enhanced chemiluminescence detection kit according to the manufacturer's instructions (Pierce Biotechnology, Rockford, IL, USA) and exposure to X-ray film (Eastman Kodak, Rochester, NY, USA). The relative band intensities in the blots were determined using Adobe
Photoshop software (Adobe Systems Inc., San Jose, CA, USA). Each experiment was performed at least three times.

Statistical analysis. All statistical analyses were performed with SPSS software (SPSS, Inc., Chicago, IL, USA) and the results are presented as the mean \pm standard error of the mean. Paired means were analyzed using Student's t-test. $\mathrm{P}<0.05$ was considered to indicate a statistically significant difference and $\mathrm{P}<0.01$ was considered to indicate a highly statistically significant difference.

\section{Results}

$N F-\kappa B$ and UCH-L1 distribution in diseased podocytes in glomerulonephritis. A previous study by our group identified that UCH-L1 expression was significantly increased in several types of immune complex-mediated glomerulonephritis (2). Therefore, the distribution of p65 was further examined using immunohistochemistry and it was compared with UCH-L1 expression in podocytes in a number of types of immune complex-mediated glomerulonephritis, including LN, IgAN and MGN. WT1 was also used as a marker of podocytes in successive sections of renal biopsy tissues (Fig. 1A-O).

In the normal kidney, WT1 is expressed in the peripheral cells of the capillary tufts and was therefore used to indicate the location of podocytes (Fig. 1A). No marked expression of $\mathrm{UCH}-\mathrm{L} 1$ and active p65 was observed in the corresponding areas (Fig. 1B-C). LN is one of the typical forms of immune complex-mediated glomerulonephritis. As shown in Fig. 1E, positive staining for UCH-L1 was increased in the glomeruli of patients with LN and was located mainly at the peripheral area of the capillary tufts in accordance with the distribution of WT1 (Fig. 1D). Activation of p65 was also increased in the glomeruli of patients with LN, with more extensive cells, including mesangial cells, endothelial cells and infiltrating leukocytes in addition to podocytes (Fig. 1F). In podocytes, the expression of UCH-L1 paralleled the activation of NF- $\mathrm{NB}$ in the glomeruli and appeared to be positively correlated in patients with $\mathrm{LN}(\mathrm{R}=0.925, \mathrm{P}<0.01$; Fig. $2 \mathrm{~A})$. As shown in Fig. 1G-L, the localization of WT1, UCH-L1 and activated p65 in patients with $\operatorname{Ig} \mathrm{AN}$ and MGN were concordant with those in patients with LN. All of these proteins were expressed in the respective podocytes. The expression of UCH-L1 also paralleled the activation of $\mathrm{NF}-\kappa \mathrm{B}$ in the glomeruli and also appeared positively correlated in $\operatorname{IgAN}(\mathrm{R}=0.927, \mathrm{P}<0.01$; Fig. $2 \mathrm{~B})$, and in MGN ( $\mathrm{R}=0.834, \mathrm{P}<0.05$; Fig. $2 \mathrm{C})$. Furthermore, the expression levels of UCH-L1 and active p65 were examined in MCD, a non-immune complex-mediated type of glomerulonephritis, in which no distinct inflammatory changes were observed in the glomeruli. Additionally, no clear expression of UCH-L1 and activated p65 was observed in podocytes in MCD (Fig. 1M and O). These results suggested that there was a close association between UCH-L1 and the activation of $\mathrm{NF}-\kappa \mathrm{B}$ in immune complex-mediated glomerulonephritis.

Upregulation of UCH-L1 expression via inflammatory stimulation with phosphorylation of $p 65$. The cultured podocytes were divided into four groups, each with or without treatment with TNF- $\alpha$, IL-1 $\beta$, LPS and ATS. Western blot analysis revealed the expression of UCH-L1 in the stimulated groups of 

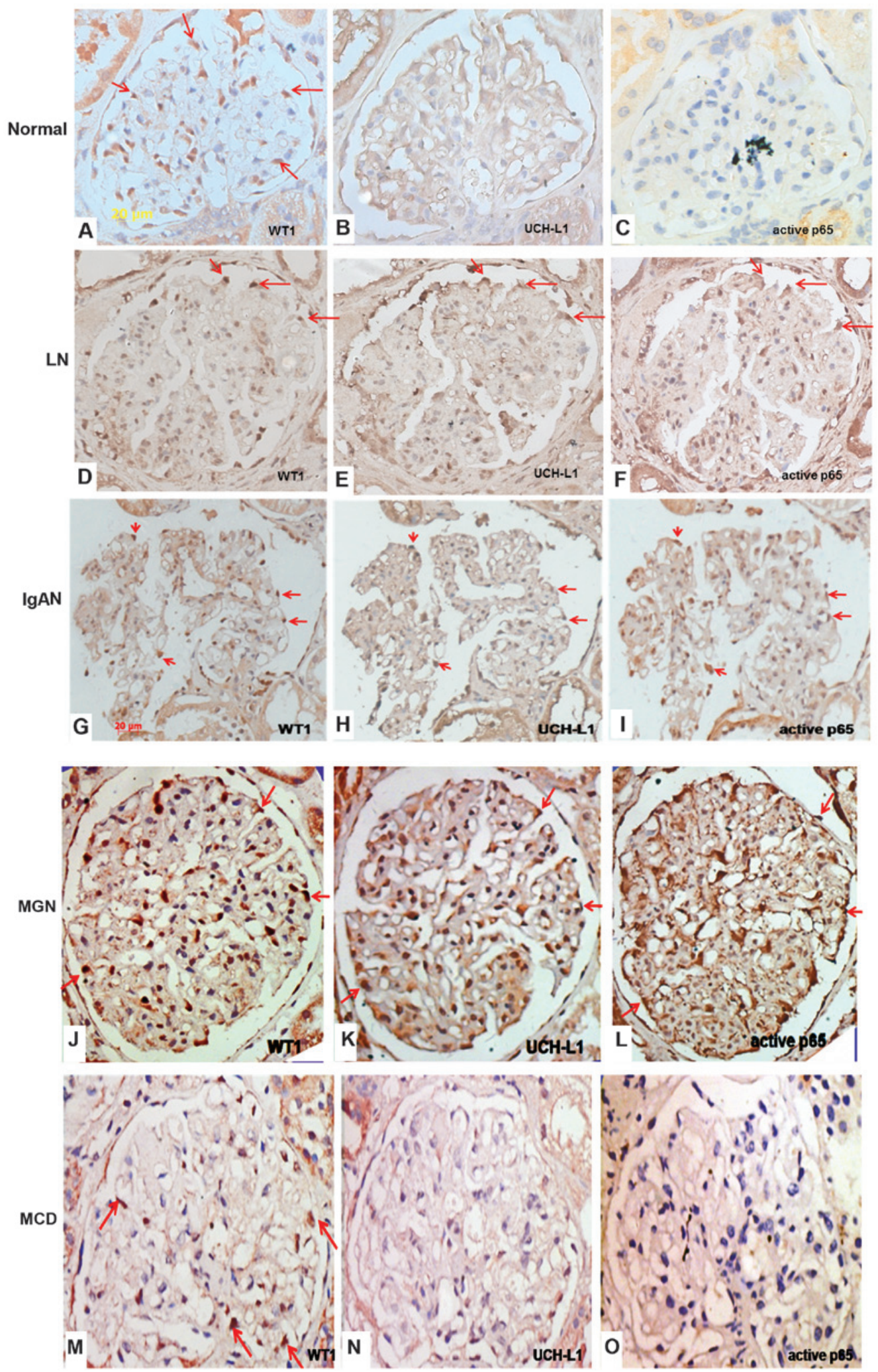

Figure 1. Expression of phosphorylated-p65 and UCH-L1 in glomeruli in several types of glomerulonephritis. (A-C) Normal controls. (A) WT1 expression indicating the location of podocytes (long arrows). (B) UCH-L1 expression was barely detectable in normal glomeruli. (C) NF- $\mathrm{KB}$ p65 activation was barely identifiable in normal glomeruli using immunostaining against activated p65. (D-F) Glomeruli from a patient with LN. (D) WT1 expression indicating the location of podocytes in LN (long arrows). (E) UCH-L1 was clearly identified in podocytes in LN (arrowheads). (F) NF- $\mathrm{kB}$ p65 activation was detectable in podocytes in LN (arrowheads). (G-I) Glomeruli from a patient with IgAN. (G) WT1 expression indicating the location of podocytes in LN (long arrows). (H) UCH-L1 expression was clearly identified in podocytes of IgAN (arrowheads). (I) NF- $\mathrm{kB}$ p65 activation was detectable in podocytes of IgAN (arrowheads). (J-L) Glomeruli from a patient with MGN. (J) WT1 expression indicating the location of podocytes (long arrows). (K) UCH-L1 expression was clearly identified in podocytes in MGN (arrowheads). (L) NF-kB p65 activation was detectable in podocytes in MGN (arrowheads). (M-O) Glomeruli from a patient with MCD. (M) WT1 expression indicating the location of podocytes in MCD (long arrows). (K) UCH-L1 expression was barely observable in podocytes in MCD. (L) NF- $\mathrm{kB}$ p65 activation was barely identifiable in podocytes in MCD. Original magnification, x400. LN, Lupus nephritis; UCH-L1, ubiquitin C-terminal hydrolase 1; IgAN, immunoglobulin A nephropathy; MGN, membranous glomerulonephritis; NF- $\mathrm{KB}$, nuclear factor- $\mathrm{\kappa B}$; MCD, minimal change disease; WT1, Wilms tumor 1. 
A

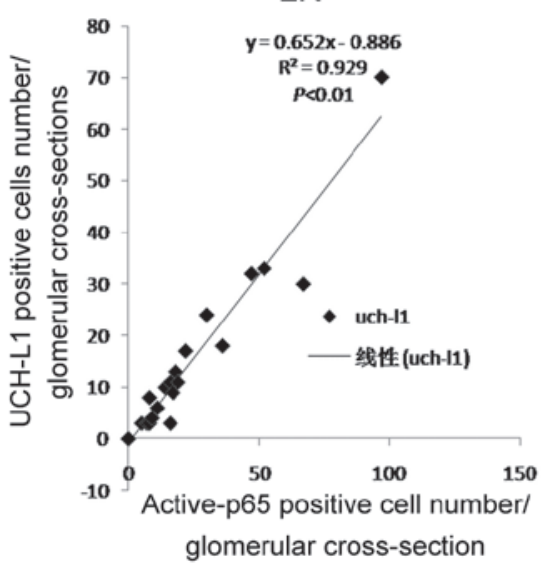

B

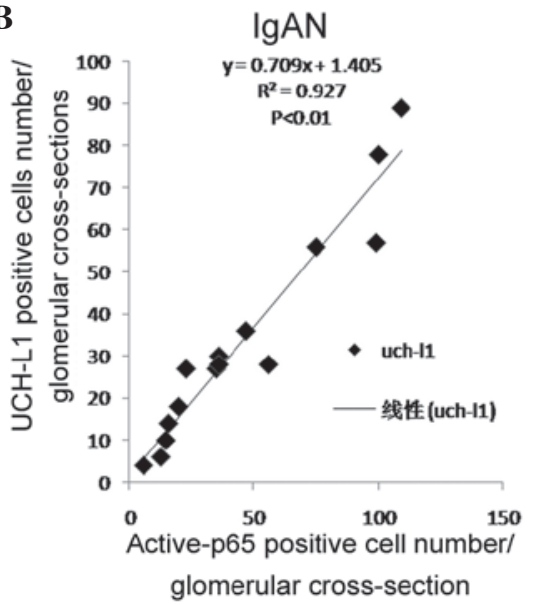

C

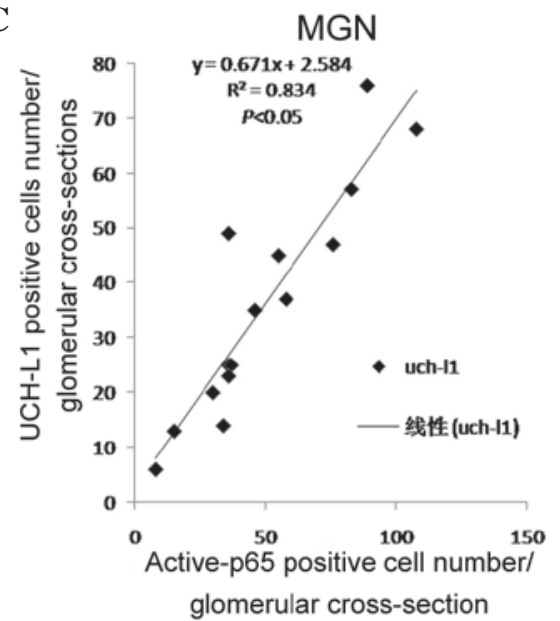

Figure 2. Statistical analysis of UCH-L1 and activated p65 in several types of glomerulonephrits. (A) Close correlation of numbers of UCH-L1-positive and p-p65-positive cells in LN. (B) Close correlation of numbers of UCH-L1-positive and p-p65-positive cells in IgAN. (C) Close correlation of the number of UCH-L1-positive and p-p65-positive cells in MGN. LN, Lupus nephritis; UCH-L1, ubiquitin C-terminal hydrolase 1; IgAN, immunoglobulin A nephropathy; MGN, membranous glomerulonephritis; p-p65, phosphorylated (active) p65.

A

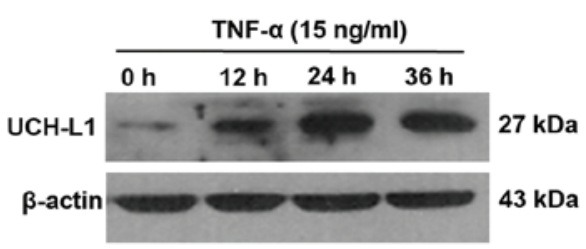

C

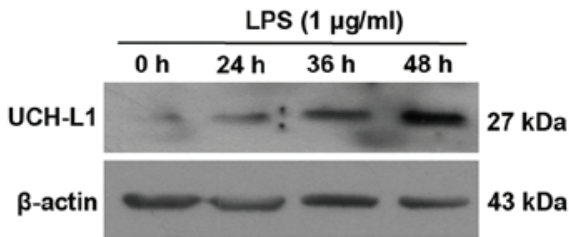

B

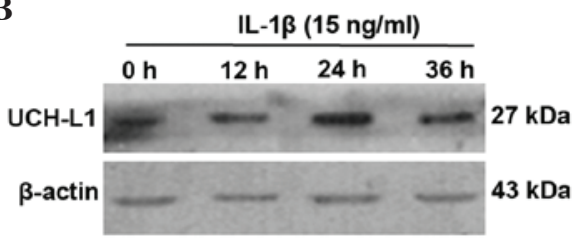

D

ATS $(50 \mu \mathrm{g} / \mathrm{ml})+$ Human serum $(30 \mu \mathrm{g} / \mathrm{ml})$

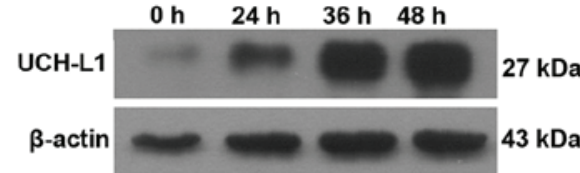

E

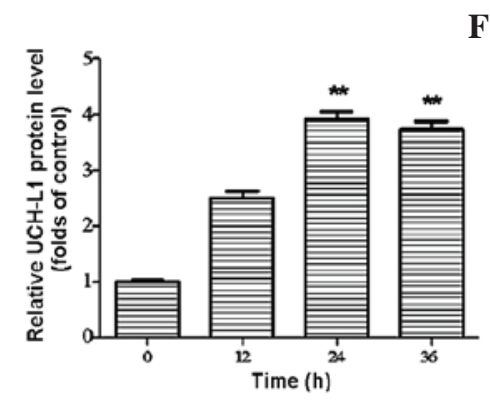

F

G

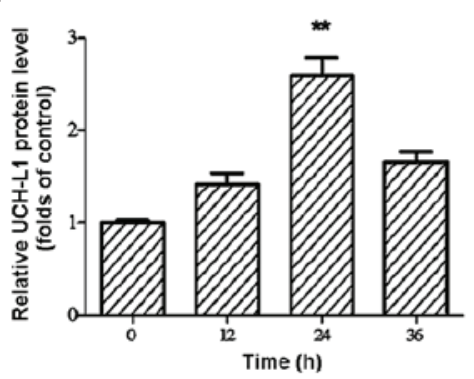

H
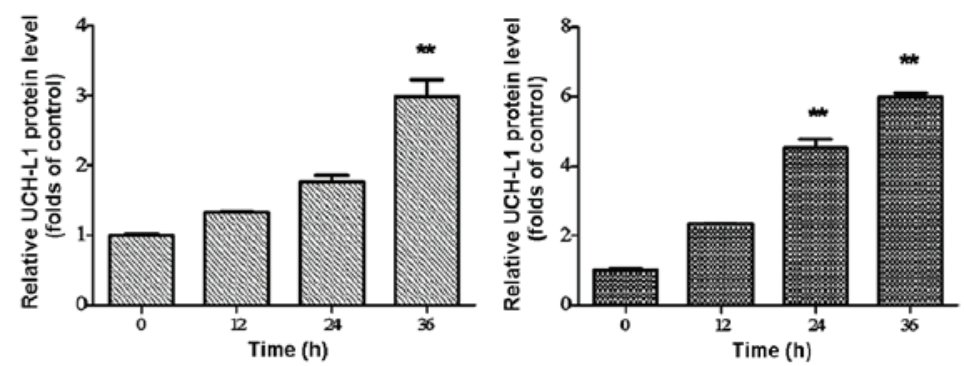

Figure 3. UCH-L1 expression in podocytes treated with TNF- $\alpha$, IL-1 $\beta$, LPS and ATS. Lysates from the podoctyes were assessed by western blot analysis using murine anti-UCH-L1 antibody. $\beta$-Actin was used as a protein loading control. Murine podocytes treated with (A) TNF- $\alpha$ (15 ng/ml), (B) IL-1 $\beta$ (15 ng/ml), (C) LPS $(1 \mu \mathrm{g} / \mathrm{ml})$ and (D) ATS $(50 \mu \mathrm{l} / \mathrm{ml})+$ human serum $(30 \mu \mathrm{l} / \mathrm{ml})$ for the indicated durations. (E-H) Relative expression of UCH-L1 quantified from the western blot analysis of A-D, respectively. Data are representative of three independent experiments. "* $\mathrm{P}<0.005$ vs. 0 h. LPS, lipopolysaccharide; UCH-L1, ubiquitin carboxy-terminal hydrolase L1; TNF, tumor necrosis factor; IL, interleukin; ATS, anti-rat thymocyte serum. 
A

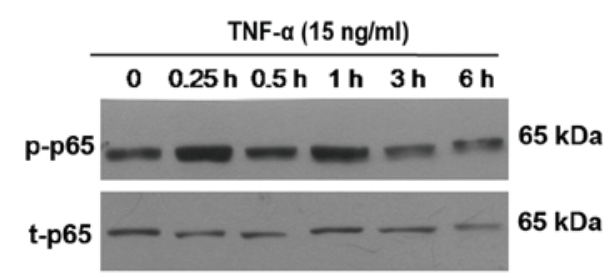

C

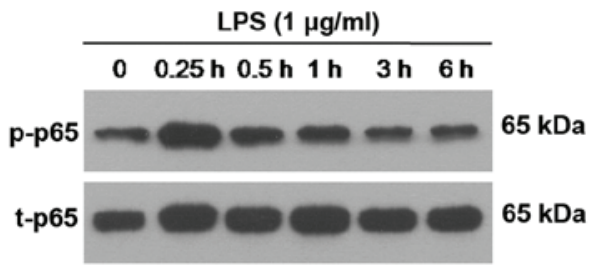

$\mathbf{E}$

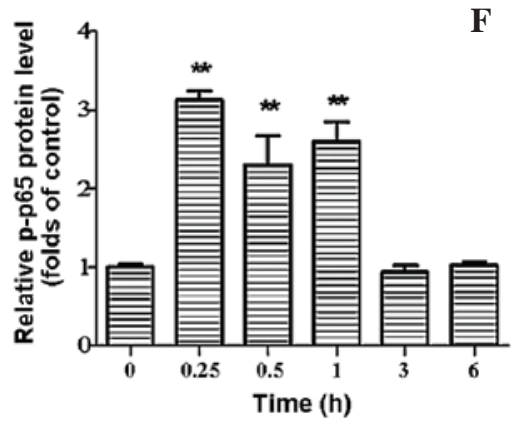

G

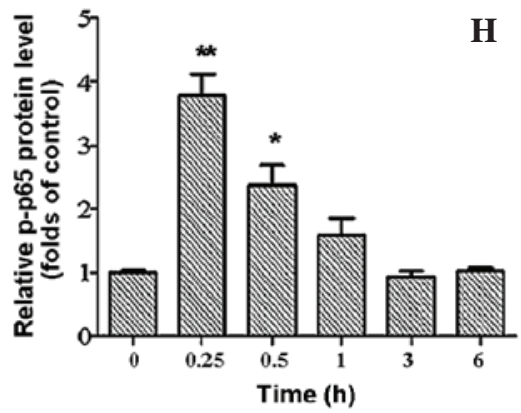

B

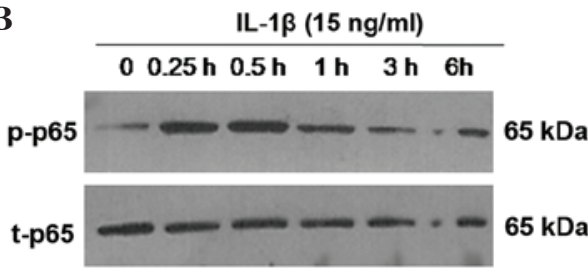

D
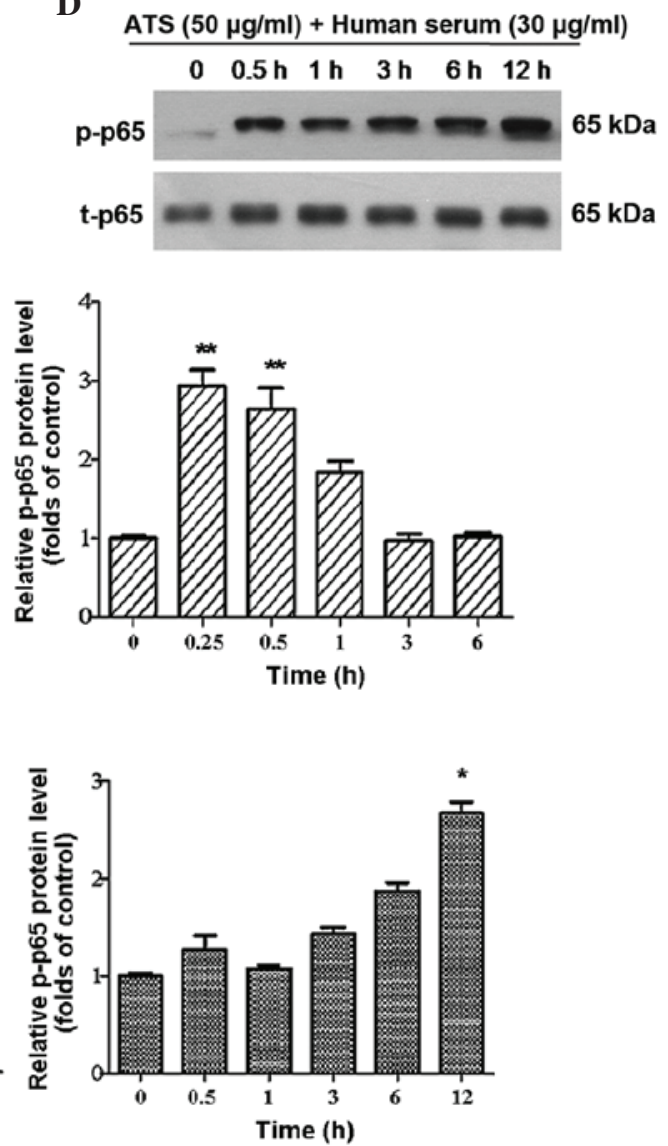

Figure 4. p-p65 expression in podocytes treated with TNF- $\alpha$, IL-1 $\beta$, LPS and ATS. Lysates from the podocytes were assessed by western blot analysis using rabbit anti-p-p65 antibody, and total-p65 was used as control for protein loading. Murine podocytes were treated with (A) TNF- $\alpha$ (15 ng/ml), (B) IL-1 $\beta$ $(15 \mathrm{ng} / \mathrm{ml})$, , C) LPS $(1 \mu \mathrm{g} / \mathrm{ml})$ and (D) ATS $(50 \mu \mathrm{l} / \mathrm{ml})+$ human serum $(30 \mu \mathrm{l} / \mathrm{ml})$ for the indicated durations. (E-H) Relative expression of p-p65 quantified from the western blot analysis of A-D, respectively. Data are representative of three independent experiments. ${ }^{*} \mathrm{P}<0.05,{ }^{* *} \mathrm{P}<0.005$ vs. 0 h.. LPS, lipopolysaccharide; TNF, tumor necrosis factor; IL, interleukin; ATS, anti-rat thymocyte serum; p, phosphorylated; $t$, total.

TNF- $\alpha$, and IL- $1 \beta$ was markedly increased in a time-dependent manner at 12 and $24 \mathrm{~h}$, reaching a maximum at $24 \mathrm{~h}$ (Fig. 3A and B). In the LPS and ATS groups, UCH-L1 expression also increased with the duration of treatment; however, the maximum level was achieved later than in the TNF- $\alpha$ and IL-1 $\beta$ groups mentioned above, namely at $48 \mathrm{~h}$. Among the stimulation groups, the highest level of UCH-L1 expression was observed in podocytes following ATS stimulation (Fig. 3C and D). These results confirmed that UCH-L1 expression in podocytes may be upregulated by pro-inflammatory mediators as well as immune stimulation.

$\mathrm{NF}-\kappa \mathrm{B}$ is a common transcription factor family consisting of different subunits, which may function as homo- or heterodimers. p65 is a well-known subunit, which is involved in the NF- $\kappa \mathrm{B}$ family in regulating the transcription of a number of genes. Phosphorylation of p65 is a typical feature in the activation of $N F-\kappa B$. Therefore, the phosphorylation of $p 65$ in the cell nucleus was further examined following treatment with TNF- $\alpha$, IL-1 $\beta$, LPS and ATS. It was identified that following treatment for $15 \mathrm{~min}$, the p65 subunit was clearly phosphorylated in cultured podocytes and the phosphorylation lasted for several hours (Fig. 4A-D). Of note, after $6 \mathrm{~h}$, the phosphorylation of p65 was greater than that in the control group. It was confirmed that the $\mathrm{NF}-\kappa \mathrm{B}$ signaling pathway was activated following stimulation with the four different treatments. Among the four treated groups, immune stimulation with ATS was able to cause the most persistent phosphorylation of p65, which lasted for at least $12 \mathrm{~h}$ and was maintained at an elevated level (Fig. 4D).

Effect of $N F-\kappa B$ inhibition on the expression of UCH-L1. Subsequently, an inhibitor of the NF- $\kappa$ B pathway was used to assess the specificity of $\mathrm{NF}-\kappa \mathrm{B}$ phosphorylation and regulation. PDTC, a specific inhibitor of $N F-\kappa B$, is capable of preventing the degradation of inhibitor of $\kappa B$ and the subsequent translocation of $\mathrm{NF}-\kappa \mathrm{B}$ from the cytoplasm into the nucleus 


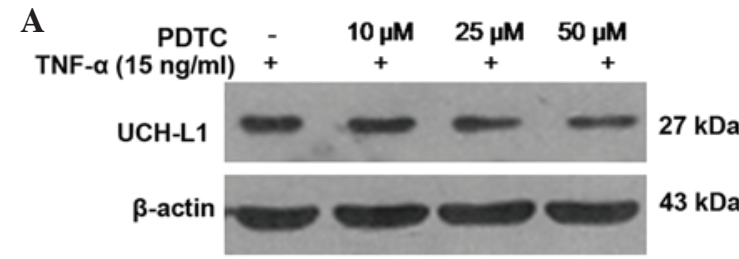

B

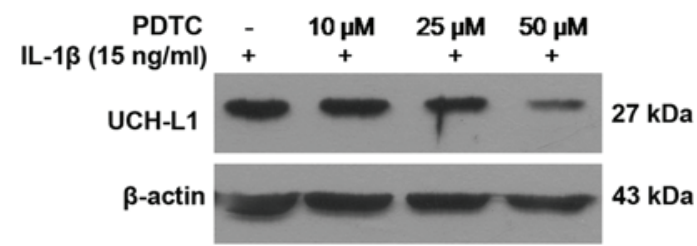

C

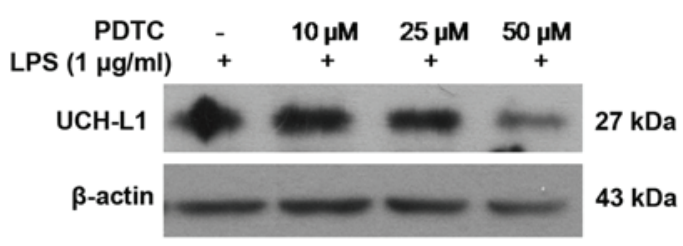

D

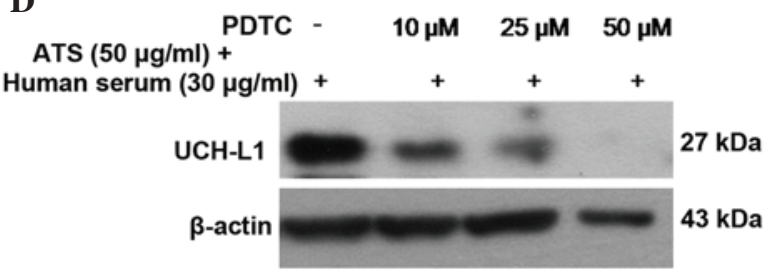

E

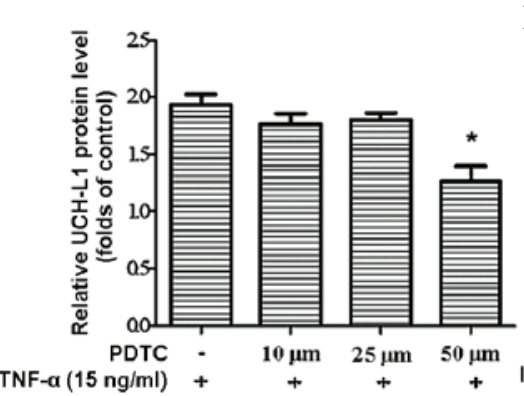

F

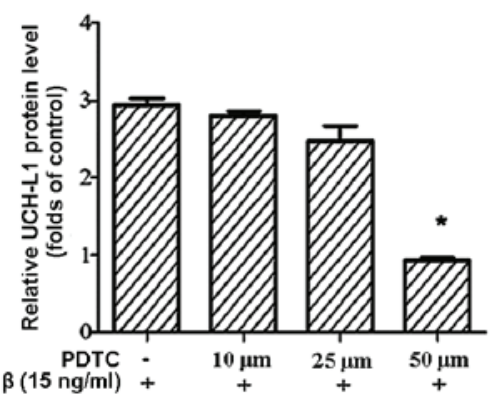

G

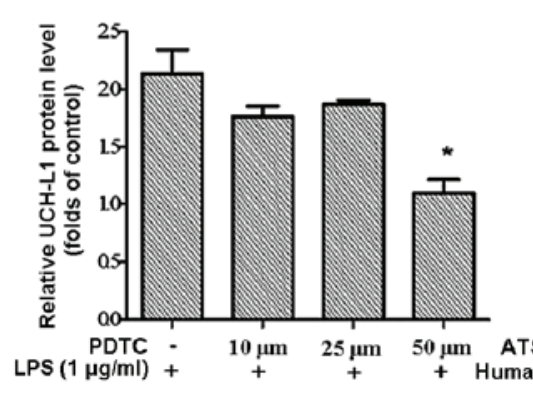

H

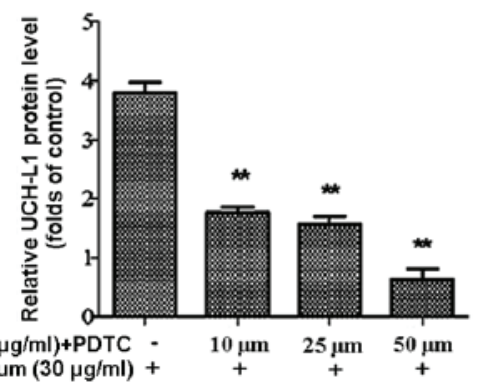

Figure 5. Inhibition of NF-kB with PDTC reduced UCH-L1 expression in podocytes treated with TNF- $\alpha$, IL-1 $\beta$, LPS and ATS. Lysates from the stimulated cells were assessed by western blot analysis using murine anti-UCH-L1 antibody, and $\beta$-actin was used as a control for protein loading. Murine podocytes were pre-treated with PDTC at the stated concentrations for $2 \mathrm{~h}$, following stimulation with (A) TNF- $\alpha(15 \mathrm{ng} / \mathrm{ml})$, (B) IL- $1 \beta$ (15 ng/ml), (C) LPS (1 $\mu \mathrm{g} / \mathrm{ml})$ and (D) ATS $(50 \mu 1 / \mathrm{ml})+$ human serum $(30 \mu \mathrm{l} / \mathrm{ml})$ for $24 \mathrm{~h}$. (E-H) Relative expression of UCH-L1 quantified from the western blot analysis of A-D, respectively. Data are representative of three independent experiments. ${ }^{*} \mathrm{P}<0.05,{ }^{* *} \mathrm{P}<0.005$, vs. PDTC. LPS, lipopolysaccharide; TNF, tumor necrosis factor; IL, interleukin; ATS, anti-rat thymocyte serum; UCH-L1, ubiquitin C-terminal hydrolase 1; PDTC, pyrrolidine dithiocarbamate.

without inhibiting other nuclear factors, including specificity protein-1, Oct and cyclic adenosine monophosphate response element-binding protein (4). In the present study, podocytes were pre-treated with PDTC at various concentrations for $2 \mathrm{~h}$ and then subjected to treatment with TNF- $\alpha$, IL-1 $\beta$, LPS and ATS for $24 \mathrm{~h}$. UCH-L1 expression significantly decreased in podocytes treated with PDTC in a dose-dependent manner (Fig. 5A-D). This result demonstrated that the inhibition of NF- $\kappa \mathrm{B}$ activation blocked the expression of UCH-L1 in response to stimulation by TNF- $\alpha$, IL- $1 \beta$, LPS and ATS.

\section{Discussion}

The present study provided further evidence supporting that UCH-L1 expression is upregulated through the activation of $\mathrm{NF}-\kappa \mathrm{B}$ in diseased podocytes in human glomerulone- phritis and in cultured mouse podocytes in vitro. It has been suggested that the NF- $\kappa \mathrm{B}$ signaling pathway is important in the underlying mechanism of podocyte injury in glomerulonephritis (28).

A study has indicated that the podocyte is a primary vulnerable cell in glomerular diseases (2). The pathological features of injured podocytes may be observed as effacement of the processes, pseudocystic changes and microvilli formation, which are closely associated with the development of proteinuria and glomerulosclerosis (29-31). In general, these podocyte changes are recognized as prominent characteristics in MCD and focal segmental glomerulosclerosis (FSGS) amongst others, which are known as non-immune complex-mediated glomerular diseases. However, it should not be ignored that severe damage to podocytes also occurs in a number of other types of glomerulonephritis, including IgAN, MGN and LN, 
via the immune injury mechanism $(1,2,25,32,33)$. Studies have demonstrated that the changes to podocyte morphology and function following injury were closely associated with the abnormalities of numerous podocyte proteins, including UCH-L1, nephrin and synaptopondin $(1,34,35)$. A previous study by our group demonstrated that $\mathrm{UCH}-\mathrm{L} 1$ is upregulated in podocytes in a number of types of immune complex-mediated glomerulonephritis, which was detected by immunoelectron microscopy, accompanied by foot process fusion (2). In addition, it was reported that the expression of UCH-L1 is associated with podocyte differentiation (25). In immature podocytes, UCH-L1 expression was higher, whereas when the cell was differentiating into a mature cell, UCH-L1 expression was significantly reduced and then eliminated, accompanied with the formation of foot processes. When the glomerular lesions formed, UCH-L1 expression increased again with foot process fusion. These results implied that elevated UCH-L1 expression is an indication of podocyte injury, which may appear in non-immune complex-mediated and immune complex-mediated glomerular diseases. However, the regulatory mechanisms of UCH-L1 or other podocyte-specific proteins are complex and remain to be elucidated.

It has been demonstrated that inflammation leads to podocyte injury involving multiple signaling pathways, including the samds, mitogen-activated protein kinase, NF- $\kappa \mathrm{B}$, $\mathrm{Wnt} / \beta$-catenin and TGF- $\beta 1$ signaling pathways $(12,29,35,36)$. $\mathrm{NF}-\mathrm{\kappa B}$ signaling is considered to be the most prominent activation pathway in the pathogenesis of human kidney diseases and numerous associated animal models $(11,37)$.

In the present study, renal biopsy sections were analyzed in numerous types of immune complex-mediated glomerulonephritis, including LN, IgAN and MGN, and the expression of active NF- $\mathrm{KB}$ p65 and UCH-L1 were identified in the podocytes. In addition, NF- $\mathrm{\kappa B}$ p65 over-activation was correlated with the expression of UCH-L1 in podocytes. By contrast, in non-immune complex-mediated glomerulonephritis, such as MCD, no marked expression of active p65 and UCH-L1 was identified in podocytes. These results indicated that immune injurious stimulation is able to increase the expression of $\mathrm{UCH}-\mathrm{L} 1$ through the NF- $\mathrm{\kappa B}$ signaling pathway, which may be vital in the pathogenesis of podocyte injury in immune complex-mediated glomerulonephritis. Furthermore, although podocyte injury and foot process effacement appeared in non-immune complex-mediated glomerulonephritis, there were no evident inflammatory changes in these glomeruli. Correspondingly, there was also no marked activation of $\mathrm{NF}-\mathrm{kB}$ and no increase of UCH-L1 in MCD. It is therefore suggested that there may be another mechanism for podocyte injury in non-immune complex-mediated glomerulonephritis.

$\mathrm{NF}-\kappa \mathrm{B}$ is known to be activated by the exposure of cells to pro-inflammatory mediators, including TNF- $\alpha$ and IL-1 $\beta(38,39)$. It is also activated by the binding of Toll-like receptors with their cognate ligands, such as LPS (4). In the present study, podocytes were therefore treated with TNF- $\alpha$, IL-1 $\beta$ and LPS in vitro, which all caused translocation of phosphorylated p65 to the nucleus and then upregulated the expression of the UCH-L1 protein. Thus, the present study further verified that $\mathrm{UCH}-\mathrm{L} 1$ is one downstream target protein of NF- $\kappa \mathrm{B}$ in podocytes. Subsequently, ATS was used in combination with human serum to treat podocytes co-cultured with rat mesangial cells, duplicating a model of immune injury. Persistent phosphorylation of p65 and a significant upregulation of UCH-L1 were identified, which were more marked than those in the groups treated with TNF- $\alpha$, IL- $1 \beta$ and LPS. This was consistent with the results of a previous study by our group (2), which demonstrated that the expression of UCH-L1 in podocytes was not elevated in non-immune complex-mediated nephritis, including MCD and FSGS. By contrast, UCH-L1 was markedly increased in immune complex-mediated glomerulonephritis (2). It has been reported that ATS is capable of interacting with antigens on the cell membrane of mesangial cells to form immune complexes and subsequently activate complements in fresh serum to assemble sublytic C5b-9, leading to immune injury to cells in vitro $(40,41)$. As podocytes present $\mathrm{Fc}$ and $\mathrm{C} 3$ receptors on the cell surface, it is possible that immune complexes and sublytic complement compounds are stimulatory factors affecting podocytes in glomeruli $(41,42)$. Therefore, the deposition of immunity compounds may be a key cause of the activation of NF- $\mathrm{kB}$ to upregulate the expression of UCH-L1 in podocytes in immune complex-mediated glomerulonephritis.

In conclusion, the present study demonstrated that in human renal biopsy samples of several forms of immune complex-mediated glomerulonephritis, the increase of $\mathrm{NF}-\kappa \mathrm{B}$ and UCH-L1 was positively correlated with diseased podocytes. However, in non-immune complex-mediated glomerulonephritis, no clear activation of NF- $\mathrm{BB}$ and no increase of UCH-L1 expression was observed. In vitro, immune stimulation also upregulated UCH-L1 through the NF- $\mathrm{KB}$ signaling pathway in mouse podocytes. These results suggested that the activation of NF- $\mathrm{kB}$ and upregulation of UCH-L1 in podocytes are vital for podocyte injury in immune complex-mediated glomerulonephritis.

\section{Acknowledgements}

The present study was supported by a grant from the National Natural Science Foundation of China (grant no. 81070566), a grant from the General Project of Weifang Medical University (grant no. K1302012) and a grant from the Science and Technology Development Plan of Medicine and Healthcare in Shandong province (grant no. 2013WS0279). The authors would like to thank Professor Hong Xu (Affiliated Children's Hospital of the Medical College of Fudan University, Shanghai, China) for her gift of the podocyte line.

\section{References}

1. Meyer-Schwesinger C, Meyer TN, Sievert H, Hoxha E, Sachs M, Klupp EM, Münster S, Balabanov S, Carrier L, Helmchen U, et al: Ubiquitin c-terminal hydrolase-11 activity induces polyubiquitin accumulation in podocytes and increases proteinuria in rat membranous nephropathy. Am J Pathol 178: 2044-2057, 2011.

2. Liu Y, Wu J, Wu H, Wang T, Gan H, Zhang X, Liu Y, Li RX, Zhao Z, Chen Q, Guo MY and Zhang Z: Uch-11 expression of podocytes in diseased glomeruli and in vitro. J Pathol 217: 642-653, 2009.

3. Greka A and Mundel P: Cell biology and pathology of podocytes. Annu Rev Physiol 74: 299-323, 2012.

4. Liu X, Ye L, Christianson G, Yang JQ, Roopenian DC and Zhu X: Nf-kappab signaling regulates functional expression of the MHC class I-related neonatal FC receptor for IgG via intronic binding sequences. J Immunol 179: 2999-3011, 2007.

5. Guijarro $\mathrm{C}$ and Egido J: Transcription factor-kB (NF-kB) and renal disease. Kidney Int 59: 415-424, 2001. 
6. Baeuerle PA: Pro-inflammatory signaling: last pieces in the NF-kappaB puzzle? Curr Biol 8: R19-R22, 1998.

7. Sakurai H, Shigemori N, Hisada Y, Ishizuka T, Kawashima K and Sugita T: Suppression of NF-kappa B and AP-1 activation by glucocorticoids in experimental glomerulonephritis in rats: molecular mechanisms of anti-nephritic action. Biochim Biophs Acta 1362: 252-262, 1997.

8. Massy ZA, Guijarro C, O'Donnell MP, Kim Y, Kashtan CE, Egido J, Kasiske BL and Keane WF: The central role of nuclear factor-kappa B in mesangial cell activation. Kidney Int (Suppl 71): 76-79, 1999.

9. Rovin BH, Dickerson JA, Tan LC and Hebert CA: activation of nuclear factor-kB correlates with MCP-1 expression by human mesangial cells. Kidney Int 48: 1263-1271, 1995.

10. Ashizawa M, Miyazaki M, Abe K, Furusu A, Isomoto $H$ Harada T, Ozono Y, Sakai H, Koji T and Kohno S: Detection of nuclear factor-kappaB in IgA nephropathy using southwestern histochemistry. Am J Kidney Dis 42: 76-86, 2003.

11. Zheng L, Sinniah R and Hsu SI: In situ glomerular expression of activated NF-kappaB in human lupus nephritis and other non-proliferative proteinuric glomerulopathy. Virchows Arch 448: 172-183, 2006.

12. Bruggeman LA, Drawz PE, Kahoud N, Lin K, Barisoni L and Nelson PJ: TNFR2 interposes the proliferative and NF-kappaB-mediated inflammatory response by podocytes to TNF-alpha. Lab Invest 91: 413-425, 2011.

13. Palanisamy N, Kannappan S and Anuradha CV: Genistein modulates NF-kappaB-associated renal inflammation, fibrosis and podocyte abnormalities in fructose-fed rats. Eur J Pharmacol 667: 355-364, 2011.

14. Mudge SJ, Paizis K, Auwardt RB, Thomas RJ and Power DA: Activation of nuclear factor-kappa B by podocytes in the autologous phase of passive heymann nephritis. Kidney Int 59: 923-931, 2001

15. Morigi M, Buelli S, Angioletti S, Zanchi C, Longaretti L, Zoja C, Galbusera M, Gastoldi S, Mundel P, Remuzzi G and Benigni A: In response to protein load podocytes reorganize cytoskeleton and modulate endothelin-1 gene: implication for permselective dysfunction of chronic nephropathies. Am J Pathol 166: 1309-1320, 2005.

16. Morigi M, Buelli S, Zanchi C, Longaretti L, Macconi D, Benigni A, Moioli D, Remuzzi G and Zoja C: Shigatoxin-induced endothelin-1 expression in cultured podocytes autocrinally mediates actin remodeling. Am J Pathol 169: 1966-1975, 2006.

17. Bondeva T, Roger $\mathrm{T}$ and Wolf $\mathrm{G}$ : Differential regulation of toll-like receptor 4 gene expression in renal cells by angiotensin II: dependency on AP1 and PU.1 transcriptional sites. Am J Nephrol 27: 308-314, 2007

18. Yu LX, Lin QX, Liao H, Feng JH, Dong XH and Ye JM: Tgf-beta1 induces podocyte injury through SMAD3-ERK-NF-kappaB pathway and FYN-dependent TRPC6 phosphorylation. Cell Physiol Biochem 26: 869-878, 2010.

19. Zhang H, Sun Y, Hu R, Luo W, Mao X, Zhao Z, Chen Q and Zhang Z: The regulation of the UCH-11 gene by transcription factor NF-kB in podocytes. Cell Signal 25: 1574-1585, 2013.

20. Fukasawa $H$ : The role of the ubiquitin-proteasome system in kidney diseases. Clin Exp Nephrol 16: 507-517, 2012.

21. Amerik AY and Hochstrasser M: Mechanism and function of deubiquitinating enzymes. Biochim Biophys Acta 1695 189-207, 2004

22. Wilson PO, Barber PC, Day IN, Thompson RJ and Polak JM: The immunolocalization of protein gene product 9.5 using rabbit polyclonal and mouse monoclonal antibodies. Br J Exp Pathol 69 : 91-104, 1988

23. D'Andrea V, Malinovsky L, Berni A, Biancari F, Biassoni L, Di Matteo FM, Corbellini L, Falvo L, Santoni F, Spyrou M and De Antoni E: The immunolocalization of PGP 9.5 in norma human kidney and renal cell carcinoma. G Chir 18: 521-524, 1997.
24. Shirato I, Asanuma K and Takeda Y: Protein gene product 9.5 is selectively localized in parietal epithelial cells of Bowman's capsule in the rat kidney. J Am Soc Nephrol 11: 2381-2386, 2000.

25. Meyer-Schwesinger C, Meyer TN, Munster S, Klug P, Saleem M, Helmchen $U$ and Stahl RA: A new role for the neuronal ubiquitin C-terminal hydrolase-11 (UCH-11) in podocyte process formation and podocyte injury in human glomerulopathies. J Pathol 217: 452-464, 2009.

26. Zhang M, Guo MY, Chen Q and M JH: The culture of rat glomerular mesangial cells. J Shanghai Med Univ: 207-209, 1995.

27. Chen GP, Guo MY and Zhang YE: Preparation of anti-thy1 serum and establishment of mesangioproliferative glomerulonephritis model in rat. J Clin Exp Pathol: 241-243, 1996.

28. Chiang ML, Hawkins EP, Berry PL, Barrish J, Hill LL: Diagnostic and prognostic significance of glomerular epithelial cell vacuolization and podocyte effacement in children with minimal lesion nephrotic syndrome and focal segmental glomerulosclerosis: An ultrastructural study. Clin Nephrol 30: 8-14, 1988.

29. Wang D, Dai C, Li Y and Liu Y: Canonical WNT/ $\beta$-catenin signaling mediates transforming growth factor- $\beta 1$-driven podocyte injury and proteinuria. Kidney Int 80: 1159-1169, 2011.

30. Ghayur A, Liu L, Kolb M, Chawla A, Lambe S, Kapoor A and Margetts PJ: Adenovirus-mediated gene transfer of TGF- $\beta 1$ to the renal glomeruli leads to proteinuria. Am J Pathol 180: 940-951, 2012.

31. Shankland SJ: The podocyte's response to injury: role in proteinuria and glomerulosclerosis. Kidney Int 69: 2131-2147, 2006

32. Borza DB, Zhang JJ, Beck LH Jr, C M and Luo W: Mouse models of membranous nephropathy: the road less travelled by. Am J Clin Exp Immunol 2: 135-145, 2013.

33. Meyer-Schwesinger C, Dehde S, Sachs M, Mathey S, Arefi K, Gatzemeier S, Balabanov S, Becker JU, Thaiss F and Meyer TN: Rho-kinase inhibition prevents proteinuria in immune-complex-mediated antipodocyte nephritis. Am J Physiol Renal Physiol 303: F1015-F1025, 2012.

34. Kato T, Mizuno S and Kamimoto M: The decreases of nephrin and nuclear WT1 in podocytes may cause albuminuria during the experimental sepsis in mice. Biomed Res 31: 363-369, 2010.

35. Greka A and Mundel P: Cell biology and pathology of podocytes. Annu Rev Physiol 74: 299-323, 2012;

36. Hirota M, Watanabe K, Hamada S, Sun Y, Strizzi L, Mancino M, Nagaoka T, Gonzales M, Seno M, Bianco C and Salomon D: SMAD2 functions as a co-activator of canonical WNT/beta-catenin signaling pathway independent of SMAD4 through histone acetyltransferase activity of p300. Cell Signal 20: 1632-1641, 2008.

37. Rangan G, Wang Y and Harris D: NF-kappaB signalling in chronic kidney disease. Front Biosci (Landmark Ed) 14: 3496-3522, 2009

38. Li Q, I, Verma M: NF- $\kappa$ B regulation in the immune system. Nat Rev Immunol 2: 725-734, 2002.

39. Schjerven H, Brandtzaeg P, Johansen FE: A novel NF- $\kappa B / R e l$ site in intron 1 cooperates with proximal promoter elements to mediate TNF- $\alpha$-induced transcription of the human polymeric Ig receptor. J Immunol 167: 6412-6420, 2001.

40. Nangaku M, Shankland SJ and Couser WG: Cellular response to injury in membranous nephropathy. J Am Soc Nephrol 16: 1195-1204, 2005.

41. Rus HG, Niculescu FI and Shin ML: Role of the c5b-9 complement complex in cell cycle and apoptosis. Immunol Rev 180: 49-55, 2001.

42. Kazatchkine MD, Fearon DT, Appay MD, Mandet C and Bariety J: Immunohistochemical study of the human glomerular c3b receptor in normal kidney and in seventy-five cases of renal diseases: loss of $\mathrm{c} 3 \mathrm{~b}$ receptor antigen in focal hyalinosis and in proliferative nephritis of systemic lupus erythematosus. J Clin Invest 69: 900-912, 1982 . 